dependent reset partially resolves this issue. One might expect that the problem of spike timing is overcome completely when considering biophysically more detailed models, such as Hodgkin-Huxley or compartment models; but even for arbitrarily refined, high-dimensional differential equation models, any reasonable time scale described must be much larger than intrinsic time scales characterizing, e.g., individual ion channels, because otherwise the very description by differential equations looses its meaning.

The study of Cessac and Viéville (2008), pushing further an alternative discrete-time view onto the world of biological neural network modeling, naturally raises more questions than it answers: in their model, discrete spike times themselves are defined arbitrarily precise (namely on the lattice) such that it remains debatable in how far the above precision problem is actually solved. More generally, how does noise affect the spike timing in networks and what is the impact of the dynamics of action potential initiation (cf. Naundorf et al., 2006)? We also need to reconsider related questions about creating (or removing) additional spikes by small perturbations and about the reliability of spikes (Jahnke et al., 2008; Teramae and Fukai, 2008). For computations in neural systems it finally seems most relevant how precisely spike times can actu- ally be detected by neurons and read out for further processing (Tiesinga et al., 2008). We definitely need to take some time to precisely think about timing before recording, simulating or analyzing the timing of action potentials in neural circuits.

\section{ACKNOWLEDGEMENTS}

This work was supported by the Federal Ministry of Education and Research (BMBF) Germany by grant number 01GQ0430 to the Bernstein Center for Computational Neuroscience (BCCN) Göttingen and by a grant of the Max Planck Society to MT. We thank L. J. Deutsch for help with manuscript preparation.

\section{REFERENCES}

Brette,R.,Rudolph,M.,Carnevale, T.,Hines, M.,Beeman, D. Bower, J.M., Diesmann, M., Morrison, A., Goodman, P. H., Harris, F. C. Jr., Zirpe, M., Natschläger, T., Pecevski, D., Ermentrout, B., Djurfeldt, M., Lansner, A., Rochel, O., Vieville, T., Muller, E., Davison, A. P., El Boustani, S., and Destexhe, A. (2007). Simulation of networks of spiking neurons: a review of tools and strategies. J. Comput. Neurosci. 23, 349-398.

Cessac, B. (2008). A discrete time neural network model with spiking neurons: rigorous results on the spontaneous dynamics. J. Math. Biol. 56, 311-345.

Cessac, B., and Viéville, T. (2008). On dynamics of integrate-and-fire neural networks with conductance based synapses. Front. Comput. Neurosci. 2, 2.

Hahnloser, R., Kozhevnikov, A., and Fee, M. (2002). An ultra-sparse code underlies the generation of neural sequences in a songbird. Nature 419, 65-70.
Jahnke, S., Memmesheimer, R.-M., and Timme, M. (2008). Stable irregular dynamics in complex neural networks. Phys. Rev. Lett. 100, 048102.

Kirst, C., Geisel, T., and Timme, M. (2009). Sequential desynchronization in networks of spiking neurons with partial reset. Phys. Rev. Lett. 102, 068101.

Naundorf, B., Wolf, F., and Volgushev, M. (2006). Unique features of action potential initiation in cortical neurons. Nature 440, 1060-1063.

Riehle, A., Grün, S., Diesmann, M., and Aertsen, A. (1996). Spike synchronization and rate modulation differentially involved in motor cortical function. Science 278, 1950-1953.

Rieke, F., Warland, D., de Ruyter van Stevenick, R., and Bialek, W. (1996). Spikes: Exploring the Neural Code. Cambridge, MIT Press.

Rokem, A., Watzl, S., Gollisch, T., Stemmler, M., Herz, A., and Samengo, I. (2006). Spike-timing precision underlies the coding efficiency of auditory receptor neurons. J. Neurophysiol. 95, 2541-2552.

Teramae,J., and Fukai, T. (2008). Temporal precision of spike response to fluctuating input in pulse-coupled networks of oscillating neurons. Phys. Rev. Lett. 101, 248105

Tiesinga, P., Fellous, J.-M., and Sejnowski, T. (2008). Regulation of spike timing in visual cortical circuits. Nat. Rev. Neurosci. 9, 97-109.

van Vreeswijk, C., and Sompolinsky, H. (1996). Chaos in neuronal networks with balanced excitatory and inhibitory activity. Science 274, 1724-1726.

Received: 24 February 2009; published: 01 May 2009 Citation: Front. Neurosci. (2009) 3,1: 2-3. doi: 10.3389/ neuro.01.009.2009

Copyright () 2009 Kirst and Timme. This is an openaccess publication subject to an exclusive license agreement between the authors and the Frontiers Research Foundation, which permits unrestricted use, distribution, and reproduction in any medium, provided the original authors and source are credited.

\title{
Cross-frequency coupling in parieto-frontal oscillatory networks during motor imagery revealed by magnetoencephalography
}

\author{
Karim Jerbi ${ }^{1,2 *}$ and Olivier Bertrand ${ }^{1,2}$ \\ 1 INSERM U821, Brain Dynamics and Cognition, Lyon, France \\ 2 University Lyon 1, Lyon, France \\ * Correspondence: karim.jerbi@inserm.fr
}

\section{A commentary on}

Interactions between posterior gamma and frontal alpha/beta oscillations during imagined actions

by Floris P. de Lange, Ole Jensen, Markus Bauer and Ivan Toni
Movement execution is the end-product of multiple intricate neural processes including action selection and planning. Although the neural dynamics involved in such internal processes are generally investigated during the build-up to movement execution, the study of motor imagery provides an alternative window on the large-scale cortical dynamics mediating formation of motor plans. Indeed, motor imagery is associated with oscillatory power modulations widely distributed in sensorimotor cortical networks (Pfurtscheller and Neuper, 1997). However, the functional role of such oscillations and the putative inter-regional coupling within and across 
multiple frequency bands are still unresolved issues.

The study by de Lange et al. (2008) addresses these timely questions by using wholehead magnetoencephalography (MEG) to investigate oscillatory brain dynamics in subjects performing a motor imagery task. The participants were required to judge the handedness of drawings of a left hand or a right hand presented at various angles. Such a task elicits internal simulations of rotating one's own hands. With frequency domain analysis and MEG source estimation, the authors evaluate modulations of various rhythmic components induced by the hand motor imagery task demands. While task-related suppressions in oscillatory power were found in the alpha $(8-12 \mathrm{~Hz})$ and beta (16-24 Hz) bands over occipitoparietal and precentral areas, significant increases in gamma-range $(50-80 \mathrm{~Hz})$ power were revealed over occipitoparietal cortex. Interestingly, when compared to right-hand motor imagery, left hand imagery was associated with stronger suppressions in contralateral motor areas. A further significant novelty of the study is the usage of cross-frequency amplitude correlation to specifically investigate oscillatory interactions between posterior parietal

\section{REFERENCES}

Bruns, A., and Eckhorn, R. (2004). Task-related coupling from high- to low-frequency signals among visual cortical areas in human subdural recordings. Int. J. Psychophysiol. 51, 97-116.

Canolty, R. T., Edwards, E., Dalal, S. S., Soltani, M., Nagarajan, S. S., Kirsch, H. E., Berger, M. S., Barbaro, N. M., and Knight, R. T. (2006). High gamma power is phase-locked to theta oscillations in human neocortex. Science 313, 1626-1628.

de Lange, F. P., Jensen, O., Bauer, M., and Toni, I. (2008). Interactions between posterior gamma and frontal alpha/beta oscillations during imagined actions. Front. Hum. Neurosci. 2, 7.

Fries, P. (2005). A mechanism for cognitive dynamics: neuronal communication through neuronal coherence. Trends Cogn. Sci. 9, 474-480.

Hummel, F. C., and Gerloff, C. (2006). Interregional long-range and short-range synchrony: a basis for complex sensorimotor processing. Prog. Brain Res. 159, 223-236.

Jensen, O., and Colgin, L. L. (2007). Cross-frequency coupling between neuronal oscillations. Trends Cogn. Sci. 11, 267-269.

Jerbi, K., Lachaux, J. P., N’Diaye, K., Pantazis, D., Leahy, R. M., Garnero, L., and Baillet, S. (2007). Coherent neural representation of hand speed in humans revealed by MEG imaging. Proc. Natl. Acad. Sci. U.S.A. 104, 7676-7681. and frontal regions during formation of a motor plan. The authors therefore provide evidence for a significant long-range anticorrelation between parietal gamma power and frontal beta power at specific periods during mental simulation of action.

Viewed in the broader context of the previous work, the findings are of particular significance. Firstly, because the findings provide novel insights into the local and long-range oscillatory dynamics within the parieto-frontal network during motor imagery, and secondly, because of the important questions raised by the findings for future research. Acknowledging the fact that behavior arises from the integrative action of large-scale brain networks (Varela et al., 2001), earlier electrophysiological studies have assessed long-range interactions between distant structures of the human brain during different experimental paradigms by using various measures of coupling (e.g., Hummel and Gerloff, 2006; Jerbi et al., 2007; Lachaux et al., 1999; Schoffelen and Gross, 2009; Sehatpour et al., 2008; von Stein et al., 2000). These studies suggest that coupling between distinct neural structures at certain frequencies might provide an efficient mechanism for inter-regional communica- tion in the brain (Fries, 2005). A growing body of research in recent years extends this view by pointing to cross-frequency coupling as a further putative mechanism mediating complex hierarchies of integrated neural ensembles at various scales (Jensen and Colgin, 2007). The study by de Lange et al. (2008) provides evidence for cross-frequency inter-areal amplitude coupling adding to a list of reported interfrequency relations such as cross-frequency phase synchrony (Palva et al., 2005) or nested oscillations. The latter findings are observed as a locking between amplitude fluctuation of faster oscillations and the phase of slower oscillations, and have been observed during active tasks as well as in spontaneous brain activity (Bruns and Eckhorn, 2004; Canolty et al., 2006; Lakatos et al., 2008; Monto et al., 2008; Mormann et al., 2005; Osipova et al., 2008; Schack et al., 2002). Finally, in order to better understand the functional role of these mechanisms, future studies will have to monitor the putative relationship between interaction measures and behavioral performance. Investigating the alteration of cross-frequency coupling in pathology will also enhance the shift from descriptions of correlations to causal inference.
Lachaux, J. P., Rodriguez, E., Martinerie, J., and Varela, F. J. (1999). Measuring phase synchrony in brain signals. Hum. Brain Mapp. 8, 194-208.

Lakatos, P., Karmos, G., Mehta, A. D., Ulbert, I., and Schroeder, C. E. (2008). Entrainment of neuronal oscillations as a mechanism of attentional selection. Science 320, 110-113.

Monto, S., Palva, S., Voipio, J., and Palva, J. M. (2008). Very slow EEG fluctuations predict the dynamics of stimulus detection and oscillation amplitudes in humans. J. Neurosci. 28, 8268-8272.

Mormann, F., Fell, J.,Axmacher, N., Weber, B., Lehnertz, K., Elger, C.E., and Fernández, G. (2005). Phase/amplitude reset and theta-gamma interaction in the human medial temporal lobe during a continuous word recognition memory task. Hippocampus 15, 890-900.

Osipova, D., Hermes, D., and Jensen, O. (2008). Gamma power is phase-locked to posterior alpha activity. PLoS ONE 3, e3990.

Palva, J. M., Palva, S., and Kaila, K. (2005). Phase synchrony among neuronal oscillations in the human cortex. J. Neurosci. 25, 3962-3972.

Pfurtscheller, G., and Neuper, C. (1997). Motor imagery activates primary sensorimotor area in humans. Neurosci. Lett. 239, 65-68.

Schack, B., Vath, N., Petsche, H., Geissler, H. G., and Möller, E. (2002). Phase-coupling of theta-gamma EEG rhythms during short-term memory processing. Int. J. Psychophysiol. 44, 143-163.
Schoffelen, J. M., and Gross, J. (2009). Source connectivity analysis with MEG and EEG. Hum Brain Mapp. (in press).

Sehatpour, P., Molholm, S., Schwartz, T.H., Mahoney, J R., Mehta, A. D., Javitt, D. C., Stanton, P. K., and Foxe, J. J. (2008). A human intracranial study of long-range oscillatory coherence across a frontaloccipital-hippocampal brain network during visual object processing. Proc. Natl. Acad. Sci. U.S.A. 105, 4399-4404.

Varela, F., Lachaux, J. P., Rodriguez, E., and Martinerie, J. (2001). The brainweb: phase synchronization and large-scale integration. Nat. Rev. Neurosci. 2, 229-239.

von Stein, A., Chiang, C., and König, P. (2000). Top-down processing mediated by interareal synchronization. Proc. Natl. Acad. Sci. U.S.A. 97, $14748-14753$.

Received: 03 March 2009; published: 01 May 2009 Citation: Front. Neurosci. (2009) 3,1: 3-4. doi: 10.3389/ neuro.01.011.2009

Copyright (c) 2009 Jerbi and Bertrand. This is an openaccess publication subject to an exclusive license agreement between the authors and the Frontiers Research Foundation, which permits unrestricted use, distribution, and reproduction in any medium, provided the original authors and source are credited. 